\title{
Analysis on Application of Probability Theory in Integral
}

\author{
Li Zhang \\ College of Mathematics and Information, China West Normal University, Nanchong, 637002, China
}

Keywords: Probability theory; integral; specific application

\begin{abstract}
The continuous random variables usually involve the probability theory and unique integral operation. Therefore, the integral operation accounts for a great proportion within the category of probability theory. Upon real calculation, students often find that the integral operation is complex and difficult and that it is hard to obtain an accurate value using the probability formula of origin. In this paper, the integral computation within the category of probability theory is discriminated and the simple and convenient computing steps are summarized. Thus, the practical advantages are highlighted, the customary integral operation is convenient and the original red tapes are reduced.
\end{abstract}

\section{Introduction}

The integral within the category of probability theory can be divided into probability integral and corresponding functional integral, in which the unique concepts of probability integral are used to check the event probability or associated difficulty and the value obtained by this operation is set as an integral. The unique object of functional integral is that the solved value is set as the function. The segmentation of probability integral covers the regularity within the category of density function, unique setting probability of certain interval, marginal distribution characterized by function, variance and concerned mathematical expectation. The discrimination of integral problem under the framework of probability theory can obtain an accurate value after operation through preset steps.

\section{Unique attribute of probability theory}

The unique thinking within the category of probability theory is to judge the setting probability characterized by something following the unique interoperability of the object and the explored cognitive law. The probability theory has such characteristics as randomness, generality and radiation; meanwhile, it also has clear directivity and can set the operation rule of different category. Specifically, the unique attributes of probability theory can be divided into the following classes:

First, the probability theory has prominent generality characteristics through segmentation. In various fields, the value of elements of random characteristics are found. The thinking extended from the probability theory characterizes these changes under framework. The relation between overall framework and quantity obtained by abstraction hides deeper association, which is abstracted into the characteristics and rules of origin. The series of characteristics encountered at ordinary times also have random characteristics. If the state of things in the surface framework is concerned purely and the unique attributes of samples are neglected, it is hard to extract the law of origin. Actually, the spacial law followed by sample setting has similar tendency. The random characteristics can be summarized as a model through abstraction, corresponding to unique probability setting.

Second, the probability theory has radiation characteristics. The progress of things often follows the associated steps and process. Correspondingly, the essential method within the framework of probability theory gradually expands from superficial theory to deep chains. For the various chains involved by probability, the law of things of origin is summarized after comparison and association and then the hidden deep law is obtained after radiation. The essential association of inherent characteristics can be found through the radiation of thinking path. In this way, the cognitive depth will be expanded horizontally, the interoperable thinking can be activated and the creativity of 
deeper layer can be found.

Third, the operation thinking under multi-level framework is created through inspection and consideration from multiple aspects. For the proposed similar problems, the deep hidden law can be found through the superficial phenomenon of surface in case of inspection from different perspective. With positive and negative comparison and unique reverse thinking, the creative new thinking can be obtained after extraction by breaking through the habitual inherent thinking modes. The new thinking under the framework of probability theory has linkage characteristics and greater span and is closely associated with the daily life. The given associated problem can be solved by creating the multi-level probability model and at the same time, the essential method relied on by the probability theory is penetrated into the daily sciences and technologies and closely links up with various subjects.

\section{Unique solving method of density function}

The commonly mentioned density function can solve the problem within the category of integral. The set density function is associated with the integrand, characterizing the variable with random characteristics. The density function under the framework of normal distribution can be used to solve this problem. The selected density function should be set as the function under the standard situation.

For example: the set function can be characterized as $\mathrm{f}(\mathrm{m})=1-\sqrt{m}$, which conforms to the origin conditions and the unique absolute value of $m$ is less than $1 . F(m)$ is regarded as piecewise function on the proposed section; thus, its characterized probability should follow the function under the piecewise situation to solve. From -2 to -1 , the value characterized by the function is zero; from 1 to 2 , the value is $m+1$; from 0 to 1 , the value is $-m+1$. For the solving of the above problems, the use of usual operation path may generate more difficulty. Therefore, by virtue of the unique geometric attributes of definite integral, the curve is located at the upper inherent position of horizontal axis and the inherent normal area of trapezoid with curve side is equivalent to the sum of area within the category of lower positions.

This law can be discriminated according to the above-mentioned description: the proposed solving probability is equivalent to the inherent area of triangle and at the same time, the area of this graph is easily obtained by observation. The continuous formula under the two-dimensional framework and the formula with random variables can obtain the above double integral through operation. Specifically, draw the inherent area of integral section and judge the type of this area to know the integral sequence; replace the double integral obtained after operation with unique quadratic integral and summarize the value characterized by upper and lower limit. Thus, the clear integral value can be obtained. During the operation, the integral area under the symmetric situation can be considered.

The origin attribute of double integral is very similar to the commonly used single integral, with recursion operation. The distribution function under the joint situation and the marginal function characterized can be replaced by the above-mentioned upper limit function. Therefore, the accurate integral within this category can be obtained if this probability is discriminated. Draw the hidden probability rule of density function and non-zero characteristics section; confirm the segmented integral category and define the clear value and section following this category.

\section{Way of proof followed by inequation}

The selected variable should have random characteristics. The preset value taking interval is set as $(o, p)$, characterizing the lower convex function under a continuous situation. If it is replaced by upper convex function, the associated variable characteristics will also change.

For example: the random variable under the continuous framework is associated with the proposed density function, $\mathrm{P}(\mathrm{m})$; the expression of this function is set as $1 / \mathrm{d}-\mathrm{c}$. If $\mathrm{m}$ is between $\mathrm{c}$ and $\mathrm{d}, \mathrm{P}(\mathrm{m})$ characterizes the above expression; if $\mathrm{m}$ is beyond this category, the unique value of 
function is set as zero. It is known from the above description that in (c, d), if the drawn function curve has the characteristics of lower convex function, the inequation value characterized by integral is set as the original value of $\mathrm{f}(\mathrm{m})$.

Actually, the direct solving is also tedious. Therefore, the clear random variable can be adopted and the unique variance of mathematical expectation is obtained after prudent operation; then the unique random value of normal distribution can be calculated by following the function attribute within the category of density function.

\section{Integral within the category of trigonometric function}

The integrand covers the trigonometric function mentioned usually, which is regarded as integral operation in a broad category. The inherent essential principles of probability theory can discriminate the unique divergence attribute of integral, thus obtaining the final value by operation.

For example: the given variable obeys the preset probability density, that is, a variable with random characteristics, and the characteristic function is set as an constant. Under this framework, the improper integral can be summarized by Euler's formula. The composition covered by integrand contains the density function under a setting situation and also obeys the original random variable.

In the above-mentioned examples, the origin theory related to the probability theory is adopted to obtain the integral within the category of trigonometric function. This indicates that the probability theory is closely associated with the unique subjects of integral, with interoperability tendency. It is known from the given examples that the probability theory can assist in solving the diversified problems within the category of algebraic analysis, embodying its convenient and unique simple advantages.

\section{Application within other categories}

(I) Characteristic continuous function

The integral characterized by probability theory also includes the unique continuous function, which is convenient to the operation within this category. For example, the fundamental basis preset by certain theorem is the definite integral under the framework of continuous function. According to the above setting rules, the given present function is changed into the continuous function. Under this situation, the final value obtained by operation is similar to the unique functional limit, but not associated with the functional value and selected division method in this period of time. Generally, a refined interval is preset for continuous equal division to select the limit value under the best circumstance. If this function is set within the given interval, the function will highlight the integrable feature. The proposed interval is replaced by short intervals after equal division. The limit value of sum formula can be obtained by selecting a point and operation.

The real operation should obey the following steps: first, conduct identical transformation of proposed sequence to obtain the integral sum of a certain form; second, check the integrand and discriminate the functional limit under the integral situation. At this time, discriminate the integrand in the equation and upper and lower limit characterized by the integral; third, change the sequence under the limit situation into the definite integral under the best situation according to the inherent integral meaning and associated integral nature. Finally, obtain the limit value in this category after operation.

For example: $\mathrm{m}(\mathrm{m}+3) ; \mathrm{m}(\mathrm{m}+6) \ldots \mathrm{m}(\mathrm{m}+3 \mathrm{~m})$, for this unique sequence, each term is of extraction of square root because the limit value under the integral situation can be obtained only by this. The solving method followed by the probability theory covers the following steps: first, obtain the inherent limit of this sum formula, including the upper and lower limit in this category; then, change the proposed expression into the common definite integral and obtain the clear limit value.

(II) Operation under continuation state

Based on the unique principle basis of probability theory, this law can be expanded: preset two functions and obtain their product by operation. Within the selected interval, this function has integrable feature. It is clear that on any segmented section, a smaller refined interval can be 
segmented. The limit sum of selected two points can be obtained after continuous operation.

For example: the selected numerical sequence covers a numeric string from $\mathrm{m} 1$ to $\mathrm{m}$. The unique quadratic sum is obtained after operation and then the sum under multiple situation is obtained by probability theory. Then, the integral results under the probability situation can be obtained by comparing the two values. If the unique integral of this sequence is needed, the unique integrable features of this sequence can be checked within the proposed section. The refined short interval includes multiple selected points. Then, the replacement tendency of numerical sequence can be predicted after the unique solving of probability theory.

(III) Law followed by integral transformation

Another theorem segmented from probability theory can be characterized as the following state: select the unique function, log, which is of clear integral features on the proposed section. Certain interval after refining is divided and then any point on this section is selected and then the common maximum value is obtained. This theorem is replaced by this form after continuous expansion: the functional value characterized by $\inf (\mathrm{c})$ has integral attribute in the change interval from 0 to a certain value. So, the inherent limit value of sequence is equal to the functional value characterized by inf(c). The above decision procedure also adopts the unique probability theory.

For example: the inherent form of a sum formula has the following features: $c+1 / c, c+2 / c \ldots c+c / c$ and the proposed formula is changed into the unique new form of $\left(1+1 / \mathrm{c}^{*} 1+2 / \mathrm{c} \ldots * 1+\mathrm{c} / \mathrm{c}\right)$. The unique value of $1 / \mathrm{c}$ power is obtained and the value of each section is obtained by probability recurrence. The integral problem related by limit can also be properly solved by probability theory. The set sum of polynomial can be replaced by the common integral.

\section{VI .Conclusion}

The probability theory is used to create the integral solving in a broad category and such solving is for segmented special form, integrand. The selected operation fails to cover the integral in the broader category. However, the unique operation path of probability theory connects the probability theory and commonly mentioned calculus orderly, expands the operation idea through flexible connection and is also convenient for cognitive communication.

\section{References}

[1] Zheng Shuhong. Application of Probability Theory in Integral[J]. Journal of Hotan Teachers College, 2007(05).

[2] Lyu Zhongqi. Application of Probability Theory in Integral[J]. Journal of Tianjin Manager College, 2009(05).

[3] Ni Dan. Application of Probability Theory in Financial Risk Theory[J]. Foreign Investment in China, 2013(10).

[4] Li Haijun. Application and Enhancement of Mean Value Theorem of Integrals[J]. The Science Education Article Collects (Late of This Month), 2007(07).

[5] Xiong Dan. Discussion on Ingenious Application of Probability Theory in Integral Computation[J]. Science \& Technology Information, 2007(09).

[6] Xia Tian. Notes on Solving Density Function of Continuous Random Variable Function by Integral-Transform Method $[\mathrm{J}]$. The Science Education Article Collects (Late of This Month), 2013(11).

[7] Liu Peng. Integral Computation in Probability Theory[J]. Journal of Chuxiong Normal University, 2007(06).

[8] Wen Chuanjun. Re-improvement of Generalized First Mean Value Theorem of Integral [J]. Studies in College Mathematics, 2011(01).

[9] Zhang Xiaomei. Research on Application of Probability Theory in Advanced Mathematics[J]. Journal of Chifeng University (Natural Science Edition), 2014(12). 